\title{
ELECTRON-ION-X-RAY SPECTROMETER SYSTEM
}

\section{OSTI}

\author{
S. H. Southworth, ${ }^{(1)}$ M. A. MacDonald, ${ }^{(2)}$ T. LeBrun, ${ }^{(3)}$ and R. D. Deslattes ${ }^{(1)}$
}

(1) National Institute of Standards and Technology, Gaithersburg, MD 20899, USA

(2) SERC Daresbury Laboratory, Warrington WA4 4AD, United Kingdom

(3)Argonne National Laboratory, Argonne, IL 60439, USA

We describe a spectrometer system developed for electron, ion, and $x$-ray spectroscopy of gas-phase atoms and molecules following inner-shell excitation by tunable synchrotron radiation. The instrumentation and experimental methods are discussed, and examples are given of electron spectra and coincidence spectra between electrons and fluorescent $x$-rays.

\section{DISCLAIMER}

\begin{abstract}
This report was prepared as an account of work sponsored by an agency of the United States Government. Neither the United States Government nor any agency thereof, nor any of their employees, makes any warranty, express or implied, or assumes any legal liability or responsibility for the accuracy, completeness, or usefulness of any information, apparatus, product, or process disclosed, or represents that its use would not infringe privately owned rights. Reference herein to any specific commercial product, process, or service by trade name, trademark, manufacturer, or otherwise does not necessarily constitute or imply its endorsement, recommendation, or favoring by the United States Government or any agency thereof. The views and opinions of authors expressed herein do not necessarily state or reflect those of the United States Government or any agency thereof.
\end{abstract}


1. Introduction

The de-excitation of an atomic inner-shell vacancy state is a complicated process due to the large number of alternative, multi-step decay pathways involving the emission of photons and electrons. These decay processes produce an array of atomic ion charge states, for example, $\mathrm{Ar}^{1+}$ to $\mathrm{Ar}^{8+}$ following Ar K-shell photoionization $[1,2]$. Molecular deexcitation processes are further complicated by dissociation resulting in fragment ions. In addition, de-excitation can depend on excitation energy, particularly near resonances and thresholds where excitation and decay must be treated as a unified process [3]. Detailed experimental information, including coincidence measurements [2], are required to characterize these complex processes.

In order to study inner-shell processes, we have developed a spectrometer system for recording electron, ion, and $x$-ray fluorescence spectra. The spectrometer has been used on beamline X-24A [4] at the National Synchrotron Light Source (NSLS) for excitation-dependent studies of Ar L-shell and K-shell photoexcitation and vacancy decay processes. In this report we describe the instrumentation and experimental methods, and examples are given of electron spectra and electron/fluorescent-x-ray coincidence spectra.

\section{Spectrometer components}

The primary components of the spectrometer system are indicated in Fig. 1. A focused, narrow-bandwidth $x$-ray beam having a high degree of linear polarization is provided by beamline $X-24 A$ [4]. This beamline is typically operated in the $X$-ray energy 
range $2-6 \mathrm{keV}$. The spectrometer contains a commercial double-pass cylindrical mirror analyzer (CMA) [5] for electron spectroscopy. The $x$-ray beam passes through an effusive beam of sample gas positioned at the source point of the CMA, and the CMA is positioned with its symmetry axis parallel to the polarization direction of the x-ray beam. The CMA accepts a large solid angle of electrons emitted into a cone of $12^{\circ}$ included angle centered about $42.3^{\circ}$ polar angle with respect to the $x$-ray polarization direction [5]. The energy resolution of the CMA is determined by the pass energy and by the use of either $1 \mathrm{~mm}$ or $4 \mathrm{~mm}$ diameter internal apertures [5].

The CMA has been used to record excitation-dependent spectra of Ar is photoelectrons, $K L L$ and $L M M$ Auger electrons, and $L_{1} L_{2,3} M$ Coster-Kronig electrons [6]. In principle, more detailed experimental information on inner-shell processes can be obtained using coincidence measurements to record a selected subset of the possible decay pathways [2]. In practice, coincidence measurements may require reduced resolution to obtain sufficient count rates. For coincidence measurements, either a timeof-flight (TOF) ion spectrometer or a $\mathrm{Si}(\mathrm{Li}) \mathrm{x}$-ray fluorescence detector is positioned opposite the CMA.

The TOF spectrometer is similar in design to that described by Hall et al. [7]. A penetrating field efficiently extracts low-energy ions from the interaction region. Ions of different mass-to-charge ratio are separated in the TOF spectrum, in which the time correlation is measured between ion pulses and photoelectrons or Auger electrons detected by the CMA. Only preliminary results have so far been obtained using this electron/ion coincidence technique. Future developments will include reversing the 
potentials on the TOF spectrometer to record threshold electrons in coincidence with fast electrons detected by the CMA.

The Si(Li) detector is a commercial $x$-ray spectrometer that has been used in combination with the CMA to record Ar is photoelectrons and Ar LMM vacancy-cascade Auger spectra in coincidence with unresolverd $A r K \alpha$ and $K B x$-ray fluorescence [8]. The data collection circuit and a coincidence spectrum are discussed in the following sections.

Phosphor screens allow viewing the position and spot size of the $x$-ray beam. Silt sizes of $1-2 \mathrm{~mm}$ are used to limit the spot size of the $x$-ray beam as it enters the spectrometer. A window having high transmission for the $x$-ray beam separates the spectrometer chamber from the ultra-high vacuum beamline. We have used a $13 \mu \mathrm{m}$ thick polypropylene film window epoxied over a $13 \mathrm{~mm}$ diameter hole drilled in a solid copper conflat-flange gasket.

The intensity of the $x$-ray beam is measured using a silicon photodiode (n on p type) located downstream of the interaction region. Such diodes are highly responsive and stable in soft $x$-ray beams [9]. The flux and the bandwidth of the $x$-ray beam depend, in part, on the acceptance apertures of the beamline, the alignment of beamline optics, and thermal and mechanical stresses on the monochromator crystals [4]. The Ar studies have been performed using Si(111) crystals in the beamline's doublecrystal monochromator. With $200 \mathrm{~mA}$ stored current in the NSLS $x$-ray ring, the $x$-ray diode typically indicates a photon flux of $6 \times 10^{10} \mathrm{sec}^{-1}$ at $3.2 \mathrm{keV}$, the Ar K-edge. The $x$-ray energy bandwidth at $3.2 \mathrm{k} \theta \mathrm{V}$ is $0.5 \cdot 0.8 \ominus \mathrm{V}$ (FWHM), based on back-reflection $x$-ray standing wave mreasurements and measurements of the Ar 1s $\rightarrow 4 p$ absorption 
resonance.

A high sample gas density in the interaction region is required to obtain sufficient count rates. An effusive gas beam is produced by a gas nozzle of $0.5 \mathrm{~mm}$ bore backed by $4 \mathrm{kPa}$ (30 torr) of sample gas, and a cryopump provides the pumping speed (1320 Vs for $\mathrm{Ar}$ ) needed to accomodate high gas fiow. The resulting background pressure in the spectrometer chamber is $1.2 \times 10^{-2} \mathrm{~Pa}\left(1.0 \times 10^{-4}\right.$ torr $)$. An automated flow control system on the gas inlet line maintains constant backing pressure behind the gas nozzle, which ensures constant gas beam density in the interaction region.

The electron count rate is quite sensitive to the positions of the gas beam and $x$-ray beam with respect to the optimum source point of the CMA. The gas nozzle is mounted on an $X-Y-Z$ manipulator to position the gas beam at the CMA's source point. $X-Y-Z$ and angular positioning of the CMA and gas nozzle with respect to the $x$-ray beam is provided by mounting the spectrometer chamber on an elevating table with a ball bearing system which allows precise positioning in the horizontal plane.

\section{CMA control and data collection circuits}

Figure 2 shows a flow chart of the circuits used for computer control of the CMA potentials and for pulse processing of detected electrons and fluorescent $x$-rays. A computer is interfaced to a CAMAC crate which contains a $0-10 \mathrm{~V}$ digital-to-analog convener (DAC) and a multichannel counter. The DAC voltage controls the potentials placed on the CMA's retarding grid and inner and outer cylinders by pairs of stable, low noise power supplies. We have used $0-1 \mathrm{kV}$ power supplies for lower-energy electron 
spectra recorded at higher resolution and $0.5 \mathrm{kV}$ power supplies for higher-energy spectra. To set the pass energy of the CMA, an offset voltage is added to the DAC voltage which controls the outer cylinder's power supply.

The observed electron kinetic energy is determined by the retarding grid potential and the pass energy. The voltages placed on the CMA's retarding grid and cylinders are measured as functions of DAC setting to derive calibration data used by the control program. This procedure gives a reproducible kinetic energy scale, indicating stable DAC and power supply circuits. However, errors in the observed kinetic energy scale can arise due to errors in the power supply calibration or due to contact potentials or small fields in the interaction region. If possible, electron peaks of known kinetic energy are recorded to check and adjust the observed kinetic energy scale.

For recording electron spectra, a commen circuit is used in which channeltron pulses are amplified, discriminated, and counted in a scaler. For coincidence measurements, however, the time characteristics of the circuits must be carefully considered to ensure that signal to noise is maximized while jitter and false coincidences are minimized. A detailed description of the circuit and methods used to record electron/x-ray-fluorescence coincidence spectra is given by LeBrun et al. [8]. Here we give a simplified description of the circuit and data analysis procedures.

Electron and $x$-ray fluorescence pulses are counted individually and sent to the stant and stop inputs of a time-to-amplitude (TAC) converter. A multichannel analyzer (MCA) displays the pulse-height spectrum from the TAC, which represents the time-correlation between detected electrons and $\mathrm{x}$-rays. A peak in the pulse-helght spectrum is observed 
due to true coincidences, that is, due to electrons and $x$-rays originating from the same atom. The peak sits atop a background due to random coincidences. Gating circuits within the MCA allow regions of the pulse-height spectrum to be selected and provide separate output pulses for true-plus-random coincidences and random-only coincidences.

The data collection program steps the DAC voltage and reads the counter to produce separate histograms of electron counts, true-plus-random coincidences, and random coincidences as functions of electron kinetic energy. After nomalization for the relative widths of the two gating regions, the random coincidence spectrum is subtracted from the true-plus-random spectrum to yield the true coincidence spectrum. This simple procedure was used to obtain the coincidence spectrum described in the next section. More elaborate data analysis procedures may be used to determine the random spectrum and reduce the noise in the resulting true coincidence spectrum $[7,8]$.

\section{Electron and coincidence spectra}

A 2000 eV electron beam provided by the CMA's intemal electron gun [5] was used to record the Ar LMM Auger spectrum plotted in Fig. 3(b). This spectrum results from direct ionization of $L$ shell electrons and reproduces the main features of the spectrum reported by Werme et al. [10]. The spectrum plotted in Fig. 3(a) was recorded using $3211.3 \mathrm{eV} \mathrm{X}$-rays at beamline $\mathrm{X}-24 \mathrm{~A}$. This excitation energy is $4.9 \mathrm{eV}$ above the $\mathrm{K}$ edge, where $\mathrm{K}$-shell ionization dominates the photoabeorption croes section. The $\mathrm{K}$ vacaney decays primarily by KLL Auger processes, leaving double vacancies in the $L$ shell. These L shell vacancies further decay by LMM processes, resulting in the spectrum of Fig. 3(a). 
Analysis of such spectra gives information on the complex arrays of states involved in the vacancy cascade pathways. Both electron spectra in Fig. 3 were recorded with the CMA operated in retarding mode at $20 \mathrm{eV}$ pass energy using $4 \mathrm{~mm}$ diameter internal apertures. The kinetic energy range $120-240 \mathrm{eV}$ was scanned using $0.1 \mathrm{eV}$ steps at an observed resolution of $0.7 \mathrm{eV}$ (FWHM). The observed kinetic energy scale was shifted by $-2 \theta \mathrm{V}$ to match the peak energies reported by Werme et al. [10].

When the Ar $\mathrm{K}$ shell is photoionized near threshold, post-collision interaction ( $\mathrm{PCl}$ ) results in energy exchanges between the 1s photoelectron and KLL Auger electron $[3,11]$. The energy distributions (lineshapes) become broadened and asymmetric, with their maxima shifted in energy. However, approximately $12 \%$ of $\mathrm{Ar} K$ vacancies decay by emission of $K \alpha$ or $K B x$-rays [12]. That fraction of 1 s photoelectrons will be unaffected by $\mathrm{PCl}$ with $\mathrm{KLL}$ Auger electrons, although PCl with LMM Auger electrons following $\mathrm{K} \alpha$ $x$-ray emission is still possible. In order to test these ideas we recorded the Ar 15 photoelectron spectrum in coincidence with unresolved $K \alpha$ and $K B x$-ray fluorescence. The results obtained using $3266.5 \mathrm{eV}$ incident $\mathrm{x}$-rays (60.1 eV above threshold) are plotted in Fig. 4. The observed energy scale has not been corrected by reference to peaks of known kinetic energies, however the relative energies and peak shapes are accurately determined. As expected, the coincidence spectrum is indeed seen to be syınmetric and its maximum is shifted to slightly higher energy in comparison with the non-coincidence spectrum. 
Acknowledgements

The measurements were made at the National Synchrotron Light Source, Brookhaven National Laboratory, which is supported by the U. S. Department of Energy, Division of Materials Sciences and Division of Chemical Sciences. This work was also supported in part by the U.S. Department of Energy Office of Basic Energy Sciences under Contract W-31-109-Eng-38. 


\section{References}

[1] T. A. Carlson and M. O. Krause, Phys. Rev. 137 (1965) A1655.

[2] J. C. Levin, C. Bledermann, N. Keller, L. Liljeby, C.-S. O, R. T. Short, I. A. Sellin, and D. W. Lindle, Phys. Rev. Lett. 65 (1990) 988.

[3] B. Crasemann, J. Phys. C9 (1987) 389; Comments At. Mol. Phys. 22 (1989) 163.

[4] P. L. Cowan, S. Brennan, R. D. Deslattes, A. Henins, T. Jach, and E. G. Kessler, Nucl. Instr. and Meth. A246 (1986) 154; P. L. Cowan, S. Brennan, T. Jach, D. W. Lindle, and B. A. Karlin, Rev. Sci. Instr. 60 (1989) 1603.

[5] P. W. Palmberg, J. Vac. Sci. Technol. 12 (1975) 379.

[6] S. H. Southworth, M. A. MacDonald, T. LeBrun, Y. Azuma, and J. W. Cooper, in preparation.

[7] R. I. Hall, A. McConkey, K. Ellis, G. Dawber, L. Avaldl, M. A. MacDonald, and G. C. KIng, Meas. Sci. Technol. 3 (1992) 316.

[8] T. LeBrun, S. H. Southworth, and M. A. MacDonald, in preparation.

[9] M. Krumrey and E. Tegeler, Rev. Sci. Instr. 63 (1992) 797.

[10] L. O. Werme, T. Bergmark, and K. Siegbahn, Phys. Scr. 8 (1973) 149.

[11] V. Schmidt, J. Phys. C9 (1987) 401.

[12] M. O. Krause, J. Phys. Chem. Ref. Data 8 (1979) 307. 
Fiqure captions

1. Diagram showing the primary components of the electron spectrometer system.

2. Flow chart of circuite used for computer control of the electron spectrometer and recording of electronffluorescent-x-ray coincidence spectra.

3. Ar LMM Auger spectra recorded using (a) a $3211.3 \mathrm{eV} x$-ray beam and (b) a $2000 \mathrm{eV}$ electron beam.

4. (a) $\operatorname{Ar}$ is photoelectron spectrum and (b) coincidence spectrum between $\mathrm{Ar}$ is photoelectrons and unresolved $K \alpha$ and $K \beta x$-ray fluorescence. The spectra were recorded simultaneously using an incident $x$-ray beam energy of $3266.5 \mathrm{eV}$. 


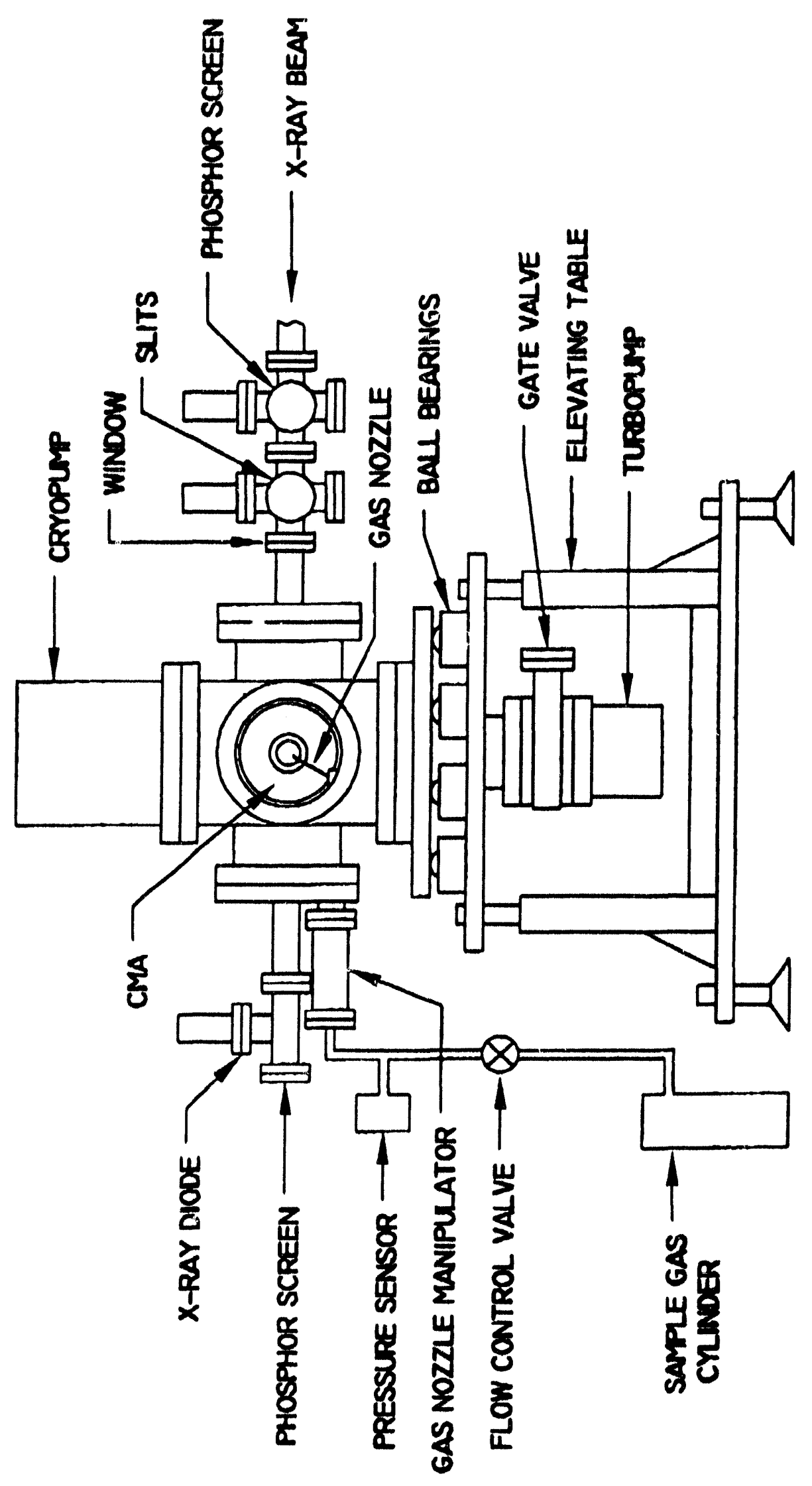

$\dot{m}$ 


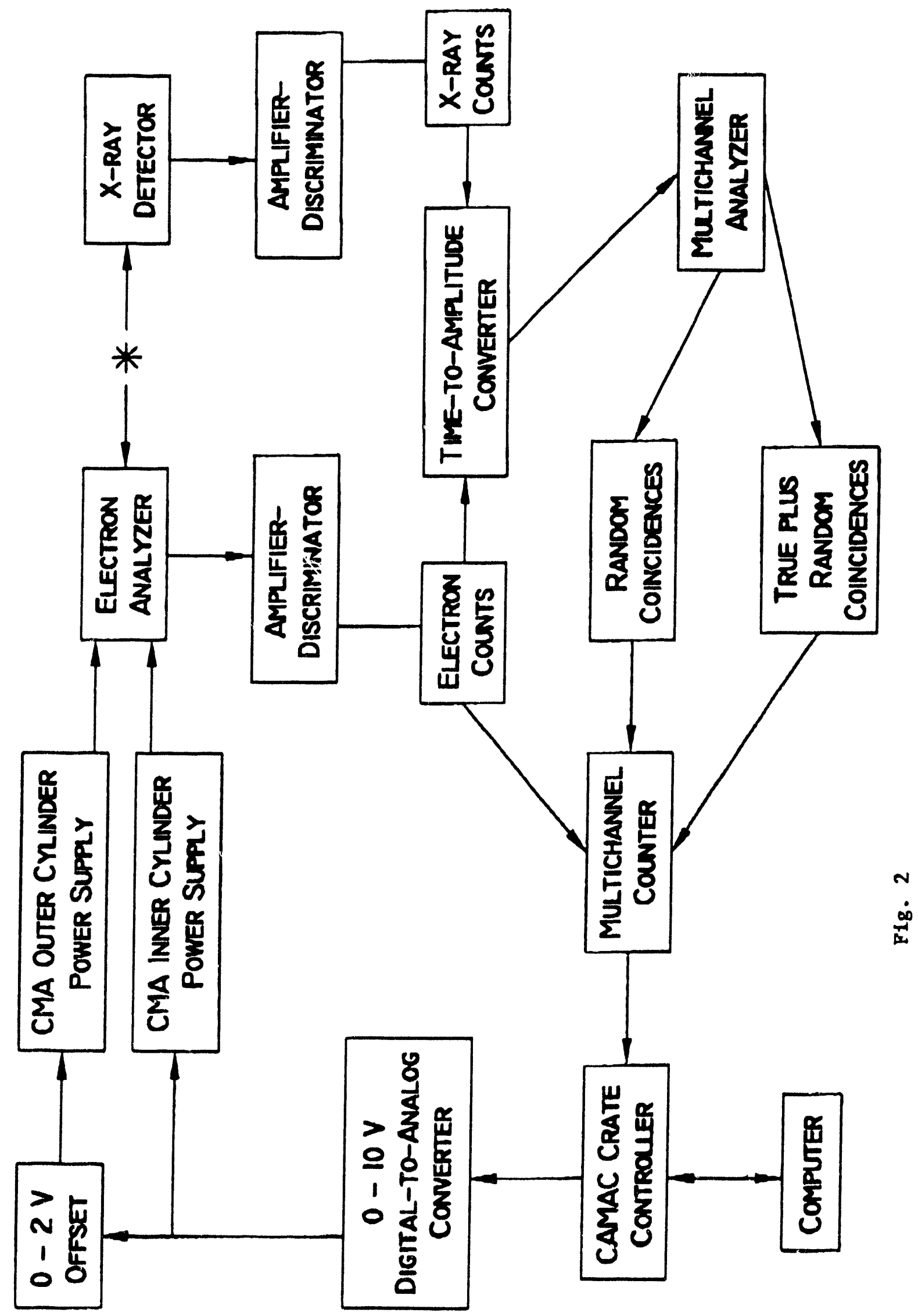




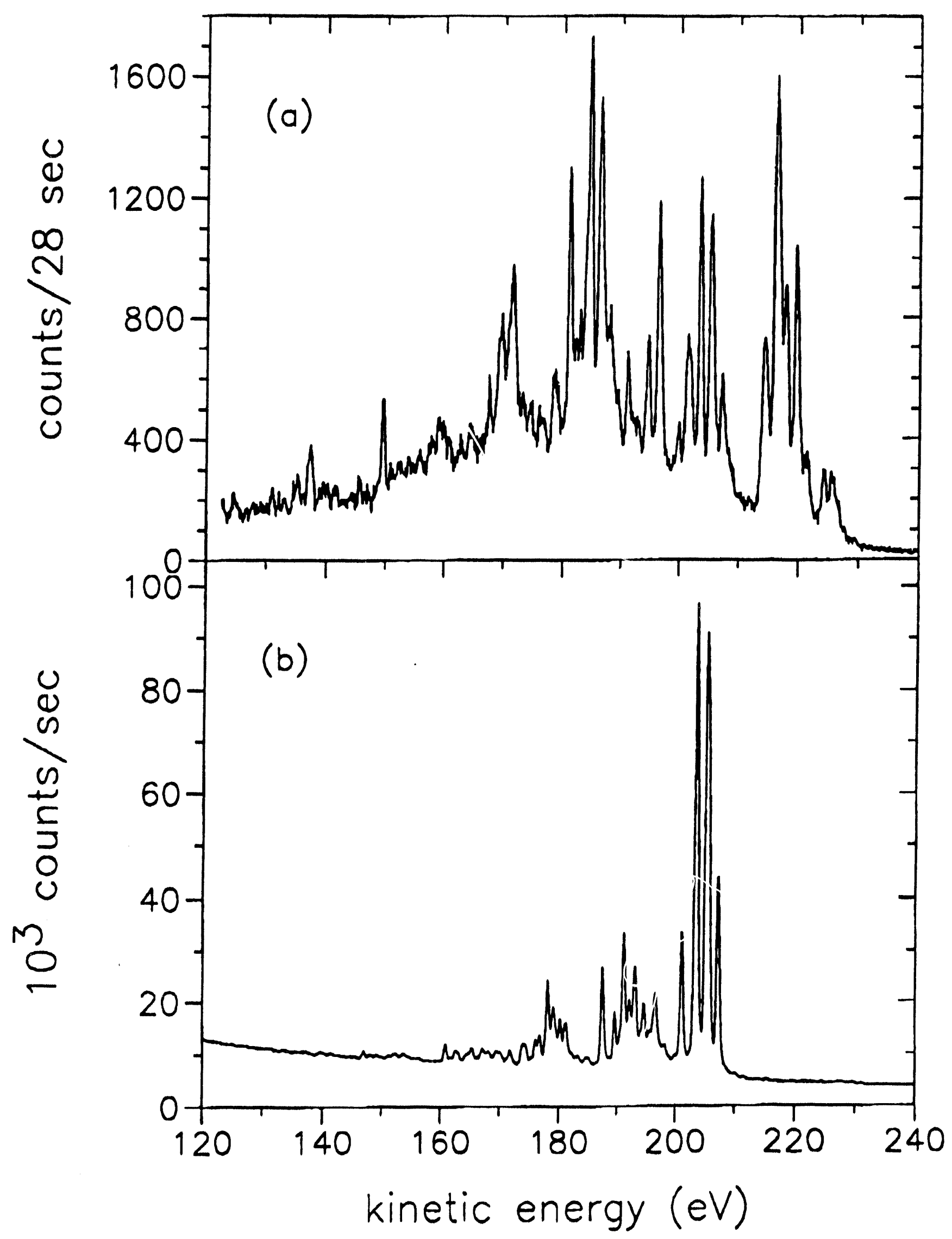

F1g. 3 


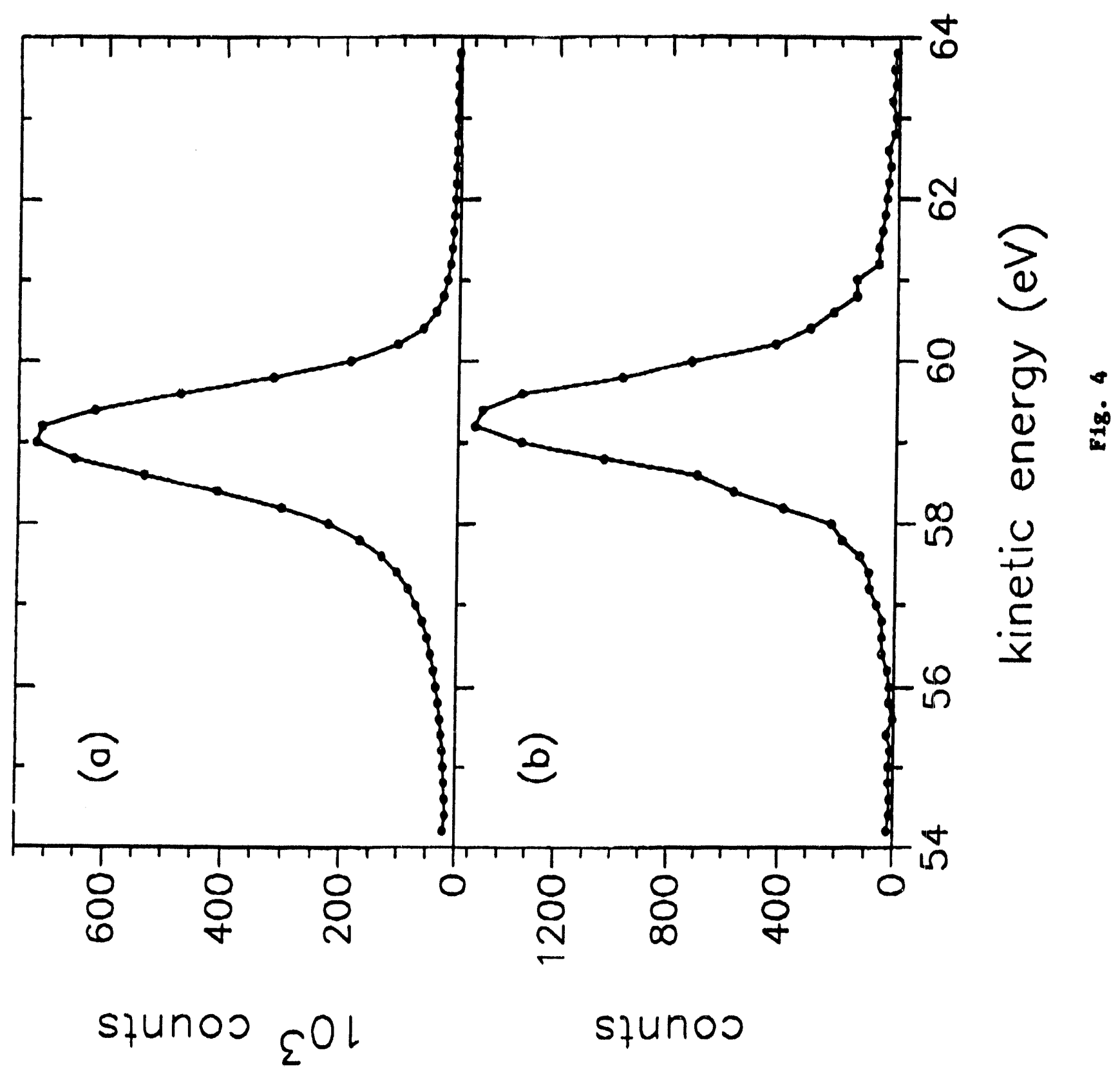



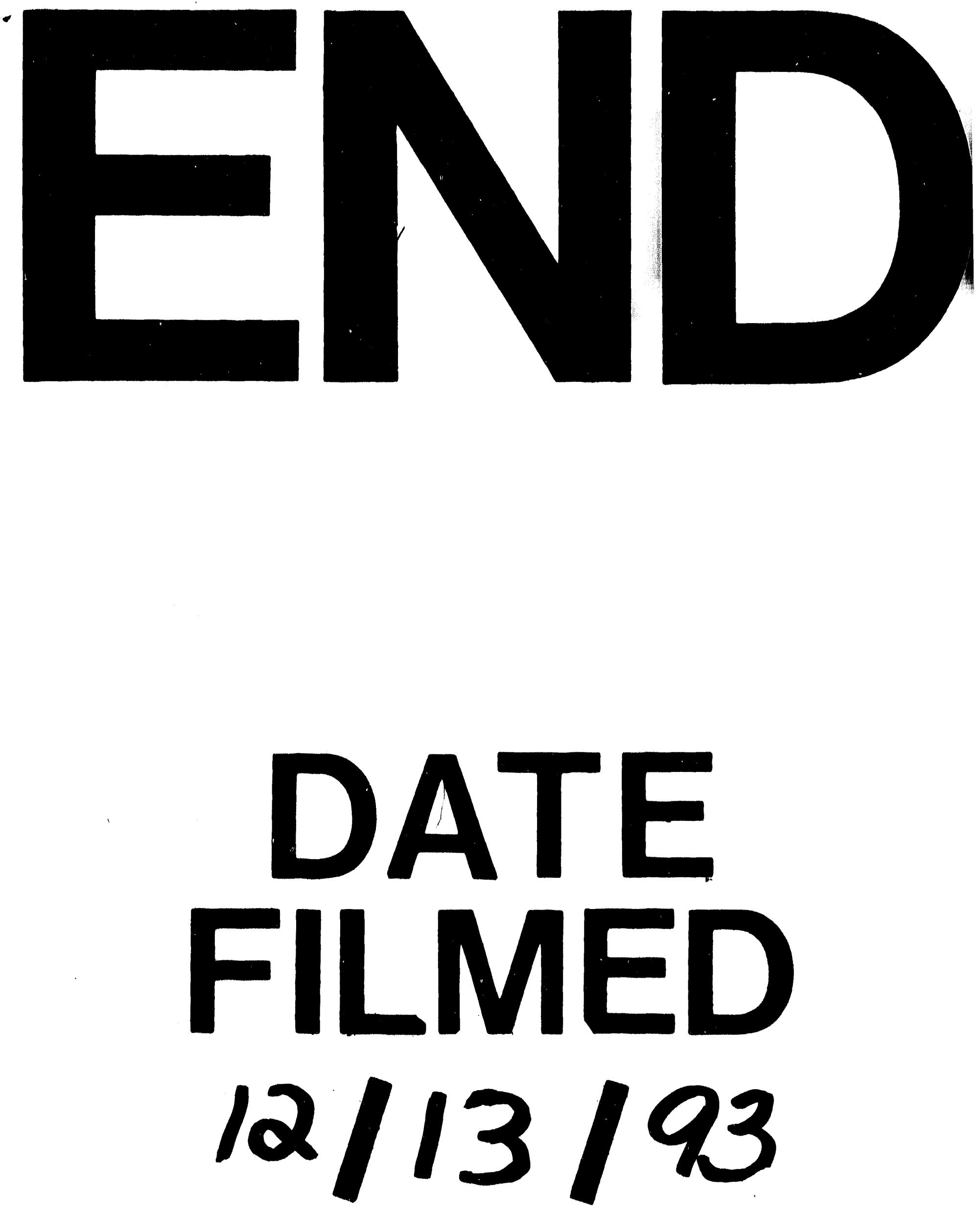
$\mid$ 